\title{
CONNECTING FINE- AND BROAD-SCALE SPECIES-AREA RELATIONSHIPS OF SOUTHEASTERN U.S. FLORA
}

\author{
Jason D. Fridley, ${ }^{1,3}$ Robert K. Peet, ${ }^{1}$ Thomas R. Wentworth,${ }^{2}$ And Peter S. White ${ }^{1}$ \\ ${ }^{1}$ Department of Biology, CB 3280, Coker Hall, University of North Carolina, \\ Chapel Hill, North Carolina 27599-3280 USA \\ ${ }^{2}$ Department of Botany, $C B$ 7612, Gardner Hall, North Carolina State University, \\ Raleigh, North Carolina 27695-7612 USA
}

\begin{abstract}
Although the rate that species accumulate with area has long been regarded as an important component of fine-scale community structure and several studies have examined this rate in meta-analyses, few if any studies have systematically examined finescale species-area relationships using a consistent survey protocol over a large region. We examined fine-scale species-area relationships using the extensive database of the Carolina Vegetation Survey (North Carolina, South Carolina, Georgia, and Tennessee, USA), including 1472 plots wherein vascular plant richness was recorded for each of six subplot sizes regularly spaced on a $\log _{10}$ scale, from 0.01 to $1000 \mathrm{~m}^{2}$. Contrary to prevailing theory, our data closely and consistently fit an Arrhenius (power law) species-area model, echoing broader-scale patterns. Species accumulation rate $(Z)$ values fell within a narrow range (95\% between 0.2 and 0.5 ) despite a 30 -fold range in $1000-\mathrm{m}^{2}$ richness. When we added regional- and global-scale richness estimates to our results, a Preston-type triphasic curve emerged. We suggest that (1) fine-scale species-area relationships are remarkably consistent and (2) full-scale species-area curves reveal scale dependencies in diversity data that are not accounted for by current species-area theory.
\end{abstract}

Key words: Arrhenius model; Carolina Vegetation Survey; Gleason model; North Carolina, USA; scale; South Carolina, USA; species accumulation rate; species-area curve; species richness.

\section{INTRODUCTION}

The sensitivity of patterns and processes to scale is of great interest in ecology and has many implications for how studies are extrapolated across space and time (Levin 1992, Crawley and Harral 2001). One of the oldest and most fundamental aspects of spatial dependence is the relationship between species richness and area, which has important implications for conservation biology and ecology (May 1994, Tilman and Lehman 1997). Since their inception, species-area curves have been the basis of debates over scale extrapolation (Gleason 1922, Arrhenius 1923), the fitting of statistical models to ecological data (Kilburn 1966, Connor and McCoy 1979, He and Legendre 1996), and the relevancy of "fine-scale" (ca. $<10000 \mathrm{~m}^{2}$ ) ecological studies to larger-scale phenomena (Williams 1964, Rosenzweig 1995, Williamson et al. 2001, Williamson 2003). In particular, there has been much contention and confusion over the relevance of species-area relationships constructed from fine-scale data to largerscale biodiversity patterns (Arrhenius 1921, 1923, Gleason 1922, 1925, Rosenzweig 1995, Williamson et al. 2001). For example, it has often been argued that fine-scale species-area curves are fundamentally dif-

Manuscript received 22 December 2003; revised 22 September 2004; accepted 15 November 2004. Corresponding Editor: T. J. Stohlgren.

${ }^{3}$ E-mail: fridley@unc.edu ferent from larger-scale curves because they are better fit by a "Gleason" (exponential) model rather than the otherwise-universal "Arrhenius" or power law model (Gleason 1922, 1925, Hopkins 1955, Whittaker 1972, van der Maarel 1988, Stohlgren et al. 1995). By extension, the processes that determine fine-scale patterns are thought to be largely decoupled from those at larger scales (Rosenzweig 1995), making problematic any extrapolation of results based on fine-scale studies, the scales at which most detailed studies of communities are performed, to landscape, regional, or global processes controlling biodiversity.

Consideration of fine-scale species-area relationships is of fundamental importance to the study of local communities. Fine-scale species-area relationships should reflect the inherent spatial structure of plant communities, and their comparison may help identify causal factors of local diversity in different systems. The rate that species accumulate with area in nested samples in community surveys has long been regarded as an important characteristic of fine-scale community structure (Arrhenius 1921, Gleason 1925, Williams 1964, Whittaker 1975). Although several studies have examined this rate, often called $Z$ value (Rosenzweig 1995), in meta-analyses of different studies conducted at different scales (see reviews in Williams [1964], Williamson [1988], and Rosenzweig [1995]), few if any studies have systematically examined variation in $Z$ values across whole regions with many independent 
samples obtained with a consistent survey protocol. Indeed, there have been very few studies with nested multi-scalar measurements of species richness in a form appropriate for evaluating true nested speciesarea curves (cf. Stohlgren et al. 1995, Kalkhan and Stohlgren 2000). As a result, there has yet to be a detailed investigation of fine-scale species-area curves using extensive empirical data, including whether they fit the Gleason or Arrhenius model and how fine-scale richness data fit within the context of larger-scale species-area relationships.

A data set particularly appropriate for addressing the nature of fine-scale species-area curves across a large geographic region is the vegetation plot database of the Carolina Vegetation Survey (CVS). Begun in 1988, the CVS database is both extensive, covering coastal to mountain habitats across the southeastern United States, and intensive, conforming to a consistent protocol of six fully nested, multi-scalar richness values for each plot from the scale of individual plants $(0.01$ $\mathrm{m}^{2}$ ) to $1000 \mathrm{~m}^{2}$. We used richness data from 1472 plots of the CVS to address the functional form and variation of fine-scale species-area curves. We specifically examined (1) the functional form for fine-scale speciesarea curves (exponential vs. power models), (2) the variation of observed species accumulation rates with area in fine-scale data ( $Z$ values) across the southeastern United States, and (3) the manner in which rates of species accumulation at fine scales compare to rates of species accumulation at larger, biogeographically significant scales.

\section{Methods}

\section{Study area and field methods}

We used 0.1-ha vegetation plots from the CVS (archived by the North Carolina Botanical Garden, Chapel Hill, North Carolina, USA) that span North Carolina and South Carolina and adjacent parts of Georgia and Tennessee (USA), from coastal dune vegetation to the mountains of the southern Blue Ridge (see Appendix, Fig. A1). The 1472 plots chosen were generally collected as regional projects, with particular concentrations in southern Blue Ridge montane forests (ca. 550 plots) and coastal plain pine savannas and woodlands (ca. 450 plots). Within a project the landscape was typically subdivided by dominant vegetation types and plots were evenly distributed across types. (Plot locations and richness data are available in the Supplement.)

All plots were surveyed with the CVS protocol described by Peet et al. (1998). Plots used in this study were sampled once between 1988 and 2000. Within each plot rooted vascular plant richness was estimated for six nested areas regularly spaced on a log-10 scale, from 0.01 to $1000 \mathrm{~m}^{2}$ (Fig. 1). Replicate richness values for areas less than $1000 \mathrm{~m}^{2}$ within each plot were av-

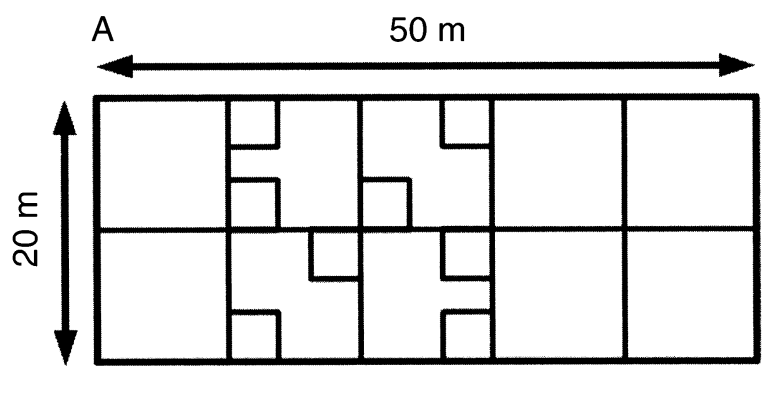

B

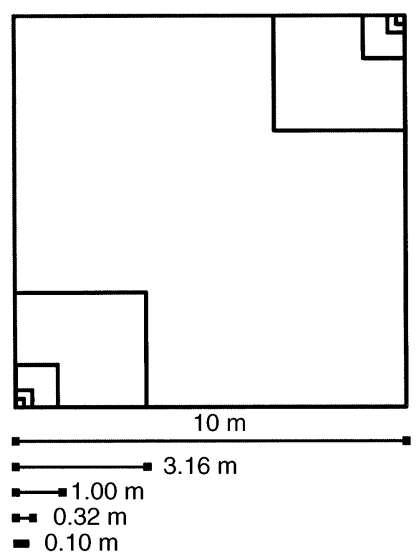

FIG. 1. Vegetation plot design of the Carolina Vegetation Survey in the southeastern United States (Peet et al. 1998). (A) The 0.1 -ha plots of $20 \times 50 \mathrm{~m}$ include 10 modules of $10 \times 10 \mathrm{~m}$, with four inner modules each containing two sets of smaller nested subplots. (B) An intensive module, $10 \times$ $10 \mathrm{~m}$, with nested subplots sized $0.01-10 \mathrm{~m}^{2}$ in opposite corners.

eraged. Vascular plant taxonomic concepts and nomenclature were standardized to follow Kartesz (1999).

\section{Analysis}

For each of 1472 plots we calculated the mean richness at six evenly spaced points along a log-10 axis of area, using eight values each at scales of $0.01,0.1,1$, and $10 \mathrm{~m}^{2}$, four at $100 \mathrm{~m}^{2}$, and one at $1000 \mathrm{~m}^{2}$. Of these 1472 plots, 108 had mean richness values of zero for the smallest subplots; because logarithms are undefined at zero, we omitted these $0.01-\mathrm{m}^{2}$ values from the analysis, but retained all other subplot values for these plots. We also retained all subplot values of zero before averaging (cf. Williams 1996). We evaluated the fit of richness $(S)$ and area $(A)$ data to Gleason semilog $(S=Z \log A+c)$ and Arrhenius $\log -\log (\log S=Z$ $\log A+\log c$ ) models using two approaches: (1) for each model, evaluation of the goodness-of-fit of the original data to a curve fit to these data by simple linear regression with richness values averaged across all 1472 plots; and (2) comparison of the distributions of Akaike Information Criterion (AIC) values for the two models when fit separately to the original data from each of the 1472 plots. AIC calculations for the Arrhenius model were based on nonlinear $\left(S=c A^{Z}\right)$ rather 

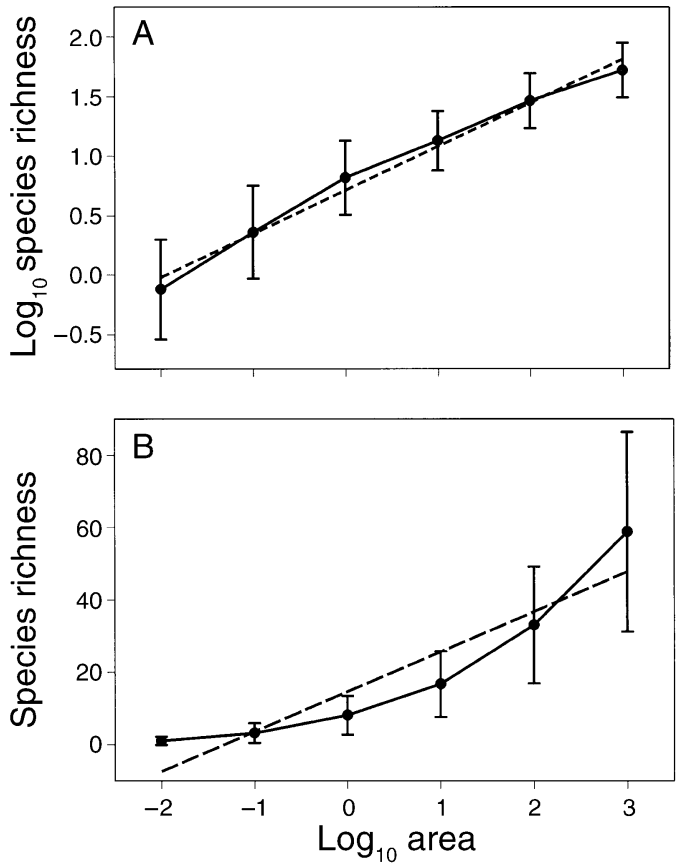

FIG. 2. Mean species richness at six points along a $\log$ scale of area for 1472 vegetation plots (area originally in units of $\left.\mathrm{m}^{2}\right)$. Solid lines connect mean values $( \pm 1 \mathrm{SD})$. Dashed lines are linear least-squares regressions and represent predicted values from (A) Arrhenius and (B) Gleason models.

than log-transformed linear model fitting to compare models of the same response units ( $S$ rather than log $S$ ). Lower AIC values indicate better goodness-of-fit. All statistical procedures were performed in S-PLUS 6.1 (Insightful Corporation, Seattle, Washington, USA). Because continental, nested species-area curves do not reach an asymptote (Williamson et al. 2001), we did not attempt to use asymptotic models that might be appropriate for other forms of species-area data $(\mathrm{He}$ and Legendre 1996, Tjorve 2003; such as islands, e.g., Lomolino [2000, 2002]).

We placed our fine-scale species-area data into the context of a larger species-area curve by increasing spatial extent from the Carolinas to a global estimate of vascular plant richness. Our global estimate of 250000 species is conservative (Govaerts 2001, Thorne 2002), but even doubling this number has no qualitative effect on our results. We included floristic richness data from the 146 counties of North and South Carolina from the USDA PLANTS database (USDANRCS 2004) and state and contiguous United States richness data from Kartesz (1999). As nonnative species comprised an extremely small part of our data set (only 296 plots contained an exotic species; only 25 plots had more than three), we included all native and nonnative taxa at all scales.

\section{RESULTS}

Plant species richness data averaged by nested quadrat sizes of areas from 0.01 to $1000 \mathrm{~m}^{2}$ for 1472 plots across the southeastern United States closely fit a power (Arrhenius) model (Fig. 2A). These same data provided a substantially poorer fit to a semi-log (Gleason) model (Fig. 2B). With untransformed richness (Gleason model), there was marked heteroscedasticity in richness among quadrat sizes (Fig. 2B); variances were relatively homogeneous when described by the Arrhenius model. Additional evaluation of the goodness-of-fit of Arrhenius and Gleason models fitted individually by plot to generate AIC distributions of 1472 values for each model suggest that a power function provides a consistently better fit to fine-scale richness data, as revealed by consistently lower AIC values (Fig. 3; $t=$ 95.1, $P<0.0001$, df $=1471)$. Together these results demonstrate the superiority of the Arrhenius model as a two-parameter function of fine-scale species-area curves for areas up to $1000 \mathrm{~m}^{2}$.

Given the power function $[\log S=Z \log A+\log$ $c$ ] as an appropriate characterization of the fine-scale species-area curve, an assessment of the rate of species accumulation $(Z)$ as area increases from 0.01 to 1000 $\mathrm{m}^{2}$ determined individually by plot reveals an approximately normal distribution with a mean of 0.372 (Fig. 4). Minimum and maximum $Z$ values are 0.120 and 0.656 ; $95 \%$ of these $Z$ values are between 0.217 and 0.538 , and half are between 0.314 and 0.427 . This relatively narrow range of species accumulation rates is found despite the fact that $0.01 \mathrm{~m}^{2}$ richness values vary from 0 to 10.5 and those of $1000 \mathrm{~m}^{2}$ plots vary from 6 to 179 .

When mean richness values for quadrat sizes (0.01 to $1000 \mathrm{~m}^{2}$ ) across our 1472 plots are put within the context of larger-scale richness values from county to global areas, a triphasic curve emerges in log-log space, with relatively fast initial and final rates of species accumulation and an intermediate region of slower accumulation (Fig. 5). Extrapolation of the fine-scale species-area relationship to the world land area gives a global richness estimate of the same order of mag-

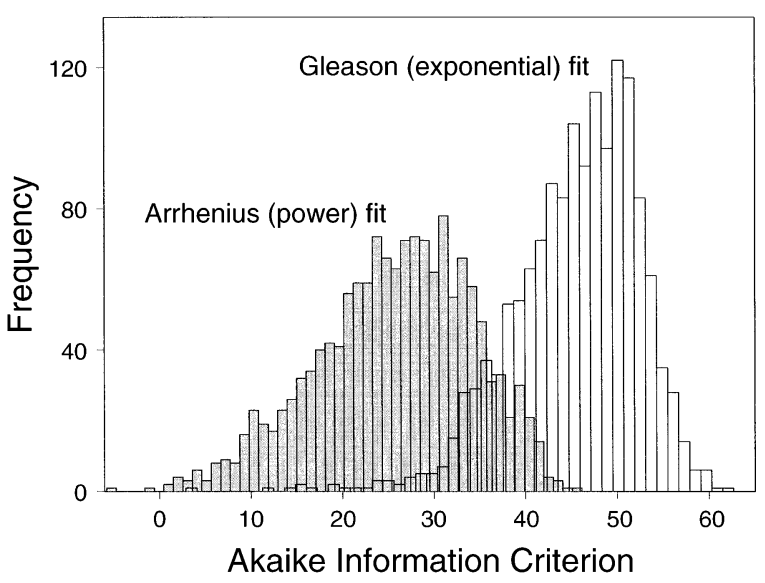

FIG. 3. Histograms of Akaike Information Criterion (AIC) values for 1472 Gleason and Arrhenius models fit to species-area data. 


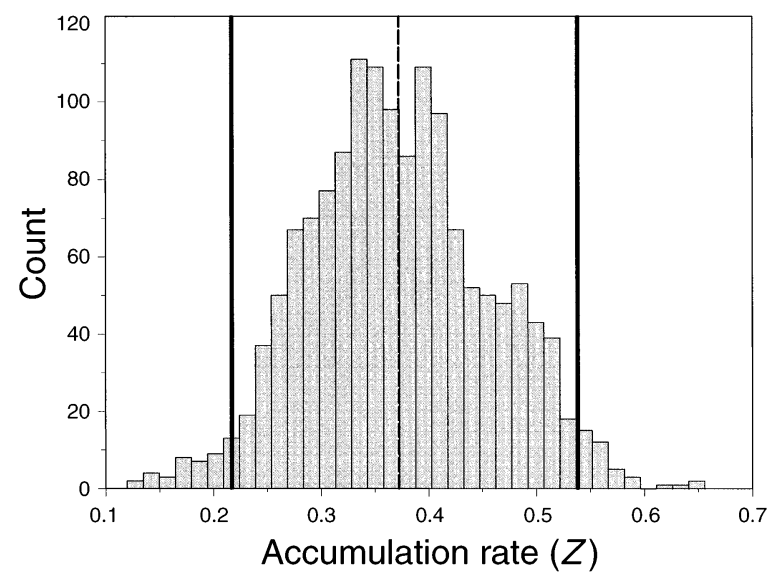

FIG. 4. Histogram of $\log -\log$ species accumulation rate $(Z)$ of 1472 vegetation plots as sample area increases from 0.01 to $1000 \mathrm{~m}^{2}$. Bin size is $\sim 0.015$. The dashed vertical line is the mean $Z$ value of 0.372 ; the solid lines $(0.217,0.538)$ delimit the central $95 \%$ of the observed $Z$ values.

nitude (ca. 850000 species) as current estimates (223 000-446000 species; see Govaerts 2001, Thorne 2002, Scotland and Wortley 2003), but areas above $1000 \mathrm{~m}^{2}$ have richness values below this line. At some area between $10^{4}$ and $10^{8} \mathrm{~m}^{2}$, the rate of species accumulation begins to steadily accelerate to reach the global richness value.

\section{DISCUSSION}

At the fine spatial scales at which communities are most often surveyed and manipulated $\left(1000 \mathrm{~m}^{2}\right.$ and below), we found that the accumulation of species richness with area closely follows an Arrhenius power law (Arrhenius 1921, 1923), the same general model that applies to species-area curves at the much larger scales of landscapes and geographic regions (Williams 1964, Rosenzweig 1995). The significance of model consistency between neighborhood and regional scales is that local investigations of species interactions and community structure can be more formally tied to patterns that occur at much larger scales. The power function is thought to be more representative of self-similarity in ecological phenomena with changes in scale (Gleason 1925, van der Maarel 1988, Rosenzweig 1995). The fit of the power function to fine-scale data thus leaves open the possibility that species richness at small scales is controlled at least in part by the same processes that control richness at larger scales.

Much previous work on fine-scale species-area curves has argued for the superiority of the Gleason exponential model, which is likely the result of insufficient data and the desire to extrapolate the fine-scale relationship to large scales without recognizing the triphasic nature of full-scale species-area curves in loglog space. Most previous empirical studies used data sets of few plots (Gleason 1925, Shmida and Whittaker 1981), few subplot sizes (He and Legendre 1996), or non-nested richness data (Rosenzweig 1995, Stohlgren et al. 1995). Over a small range of scales the relative goodness-of-fit of power and exponential functions is difficult to determine, particularly with few data points (He and Legendre 1996). Gleason (1922, 1925) introduced and defended the exponential species-area model primarily on the basis of its more reasonable approximation of richness at much larger scales than the plant neighborhood. We agree with Gleason that finescale power curves over-predict richness for larger areas (Fig. 5). However, this is not a result of the power function being a poor fit to fine-scale data, but rather that $\log -\log$ species-area curves are triphasic, with slower accumulation rates in intermediate areas. In his comprehensive treatment of species-area curves, Rosenzweig (1995) dismissed the usefulness of fine-scale species-area relations as a result of several of the above concerns. Following Preston (1960), we assert that the difference in rate of species accumulation at fine scales

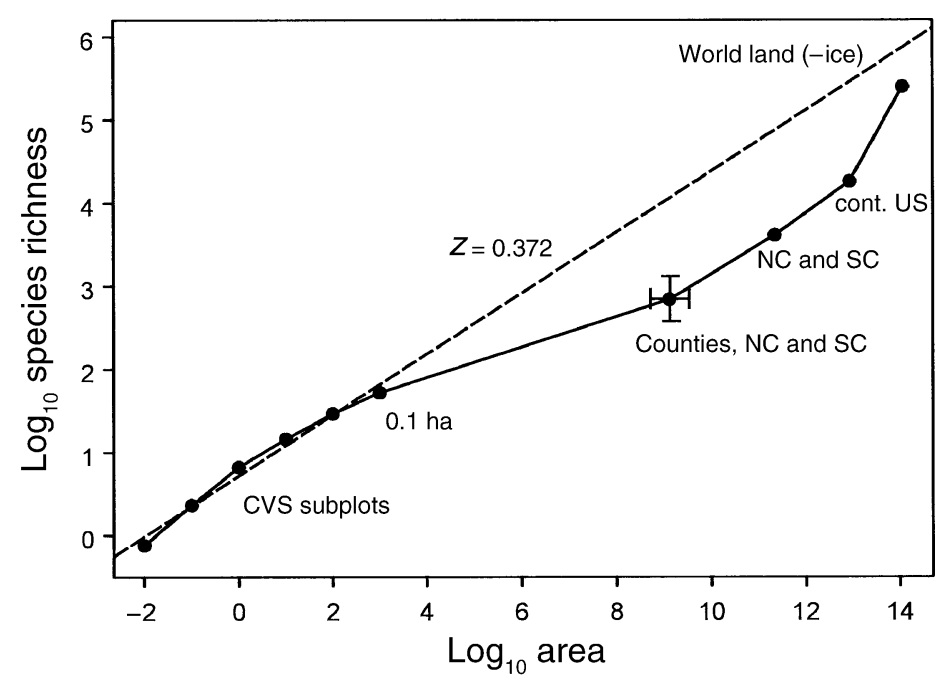

FIG. 5. Log-log species-area curve (Arrhenius curve) of vascular plant richness from fine scales to global land area, starting from within the Carolinas, USA (area originally in units of $\mathrm{m}^{2}$ ). The lowest six values are mean richness values from 1472 0.1-ha vegetation plots of the Carolina Vegetation Survey (CVS), with the dashed line representing the mean species accumulation rate for these scales. Richness data (mean and 95\% CI) for the 146 counties of North Carolina (NC) and South Carolina (SC) pooled are from the USDA PLANTS database (USDANRCS 2004). State and contiguous (cont.) United States richness data are from Kartesz (1999); global richness (world land - ice) is an estimate of 250000 species. 
compared to intermediate scales is an important consideration in scaling up from local to regional studies, but the consistency of fine-scale curves and their general agreement with a power law attests to their significance as a tool for analyzing diversity patterns.

We found rates of species accumulation ( $Z$ values) at fine scales to be consistently higher than those generally reported for intermediate scales (Fig. 5; Preston 1960, Williams 1964, Williamson 1988, Rosenzweig 1995) and of surprisingly narrow distribution, with half in the range of 0.32 and 0.43 , despite large variation in richness at all scales. Preliminary evidence suggests $Z$ values in this range are typical for multi-scale floristic studies at the scales of $1000 \mathrm{~m}^{2}$ and below (Shmida and Wilson 1985; Oklahoma tallgrass prairie, M. W. Palmer, personal communication; cf. Crawley and Harral 2001, Williamson 2003). This consistency suggests some statistical or biological constraint over the spatial distribution of species richness at small scales that deserves further study. In particular, fine-scale richness should be fundamentally constrained by the density of individuals in small quadrats, what Preston (1960) referred to as "sampling error" (not errors resulting from varied survey effort at different scales). Although the influence of this sampling error can be estimated with a variety of rarefaction techniques if individual density data are available for each scale (Preston 1960, Gotelli and Colwell 2001), estimating individual density for organisms of indeterminate growth is notoriously difficult and in many cases impossible (e.g., clonal herbs). Separation of sampling and habitat processes in the control of fine-scale species-area curves of plant communities remains a critical issue for further research.

Of the relatively few existing species-area curves that include nested data from the scale of individuals to that of national or global richness for a taxocene, nearly all are conspicuously triphasic, with fast initial and final rates of species accumulation and an intermediate region of slow accumulation (Preston 1960, Williams 1964, Shmida and Wilson 1985, Hubbell 2001; but see Crawley and Harral [2001]). The ubiquity of the three phases of full-scale species-area curves suggests that this should be the standard species-area model (Hubbell 2001, Williamson et al. 2001, Williamson 2003), replacing the two-phase intra- vs. interprovincial model suggested by Rosenzweig (1995). Shmida and Wilson (1985), Rosenzweig (1995), and Hubbell (2001), among others, have suggested that the upturn in the species-area curve approximates the scale at which large barriers to the dispersal of entire biotas lead to over-enrichment of species richness from the production of ecological equivalents in isolated biotic provinces. We found that this upturn occurs at or possibly below the scale of a county (ca. $10^{9} \mathrm{~m}^{2}$ ) within the Carolinas (Fig. 5), a much smaller scale than what most researchers would call a biotic province. This may indicate that limits to the dispersal of plant taxa are expressed at smaller distances than previously recog- nized, and we suggest more detailed studies of this point of upturn in full-scale species-area curves from other regions. For example, we hypothesize that this point of upturn occurs at larger areas in regions of higher latitude, following Rapoport's rule (Rapoport 1982) that geographic range sizes increase with latitude.

Our results highlight the consistency of fine-scale species-area curves and suggest consistency with larger-scale biogeographic patterns (Preston 1960). We see future research needed to document the relationship between fine-scale and regional/global diversity species-area curves. Of particular importance is identification of the principal biological or statistical constraints on species accumulation rates at different spatial scales, including the influence of density constraints on richness at various scales.

\section{ACKNOWLEDGMENTS}

We gratefully acknowledge the $>600$ individuals who have participated in collection of the Carolina Vegetation Survey data set, and particularly A. Weakley and M. Shafale. J. Weiss provided statistical advice and members of the UNC Plant Ecology Laboratory, and especially J. Gramling, D. Vandermast, T. Jobe, A. Senft, M. McKnight, and J. Kaplan, provided valuable suggestions at all stages of this work. Plot inventory was made possible through challenge cost-share grants from the United States Forest Service. Data compilation and analysis was supported by NSF grants DBI-9905838 and DBI0213794 to R. K. Peet. J. D. Fridley was supported by the National Parks Ecological Research Fellowship program, a partnership between the National Park Service, the Ecological Society of America, and the National Park Foundation and funded through a generous grant from the Andrew W. Mellon Foundation.

\section{Literature Cited}

Arrhenius, O. 1921. Species and area. Journal of Ecology 9: 95-99.

Arrhenius, O. 1923. On the relation between species and area-a reply. Ecology 4:90-91.

Connor, E. F., and E. D. McCoy. 1979. The statistics and biology of the species-area relationship. American Naturalist 113: 791-833.

Crawley, M. J., and J. E. Harral. 2001. Scale dependence in plant biodiversity. Science 291:864-868.

Gleason, H. A. 1922. On the relation between species and area. Ecology 3:158-162.

Gleason, H. A. 1925. Species and area. Ecology 6:66-74

Gotelli, N. J., and R. K. Colwell. 2001. Quantifying biodiversity: procedures and pitfalls in the measurement and comparison of species richness. Ecology Letters 4:379391.

Govaerts, R. 2001. How many species of seed plants are there? Taxon 50:1085-1090.

He, F., and P. Legendre. 1996. On species-area relations. American Naturalist 148:719-737.

Hopkins, B. 1955. The species-area relations of plant communities. Journal of Ecology 43:409-426.

Hubbell, S. P. 2001. The unified neutral theory of biodiversity and biogeography. Princeton University Press, Princeton, New Jersey, USA.

Kalkhan, M. A., and T. J. Stohlgren. 2000. Using multi-scale sampling and spatial cross-correlation to investigate patterns of plant species richness. Environmental Monitoring and Assessment 64:591-605. 
Kartesz, J. T. 1999. A synonymized checklist and atlas with biological attributes for the vascular flora of the United States, Canada, and Greenland. First edition. In J. T. Kartesz and C. A. Meacham, editors. Synthesis of the North American flora. Version 1.0. North Carolina Botanical Garden, Chapel Hill, North Carolina, USA.

Kilburn, P. 1966. Analysis of the species-area relation. Ecology 47:831-843.

Levin, S. A. 1992. The problem of pattern and scale in ecology. Ecology 73:1943-1967.

Lomolino, M. V. 2000. Ecology's most general, yet protean pattern: the species-area relationship. Journal of Biogeography 27:17-26.

Lomolino, M. V. 2002. The species-area relationship does not have an asymptote-comment. Journal of Biogeography 29:555-557.

May, R. M. 1994. Ecological science and the management of protected areas. Biodiversity and Conservation 3:437448.

Peet, R. K., T. R. Wentworth, and P. S. White. 1998. A flexible, multipurpose method for recording vegetation composition and structure. Castanea 63:262-274.

Preston, F. W. 1960. Time and space and the variation of species. Ecology 41:611-627.

Rapoport, E. H. 1982. Areography: geographical strategies of species. Pergamon Press, New York, New York, USA.

Rosenzweig, M. L. 1995. Species diversity in space and time. Cambridge University Press, Cambridge, UK.

Scotland, R. W., and A. H. Wortley. 2003. How many species of seed plants are there? Taxon 52:101-104.

Shmida, A., and R. H. Whittaker. 1981. Pattern and biological microsite effects in two shrub communities, southern California. Ecology 62:234-251.

Shmida, A., and M. V. Wilson. 1985. Biological determinants of species diversity. Journal of Biogeography 12:1-20.

Stohlgren, T. J., M. B. Falkner, and L. D. Schell. 1995. A modified-Whittaker nested vegetation sampling method. Vegetatio 117:113-121.
Thorne, R. F. 2002. How many species of seed plants are there? Taxon 51:511-512.

Tilman, D., and C. L. Lehman. 1997. Habitat destruction and species extinctions. Pages 233-249 in D. Tilman and P. Kareiva, editors. Spatial ecology: the role of space in population dynamics and interspecific interactions. Princeton University Press, Princeton, New Jersey, USA.

Tjorve, E. 2003. Shapes and functions of species-area curves: a review of possible models. Journal of Biogeography 30:827-836.

USDA-NRCS (Natural Resources Conservation Service). 2004. The PLANTS database, version 3.5. National Plant Data Center, Baton Rouge, Louisiana, USA. 〈http:// plants.usda.gov $\rangle$

van der Maarel, E. 1988. Species diversity in plant communities in relation to structure and dynamics. Pages 1-14 in H. J. During, M. J. A. Werger, and H. J. Willems, editors. Diversity and pattern in plant communities. SPB Academic, The Hague, The Netherlands.

Whittaker, R. H. 1972. Evolution and measurement of species diversity. Taxon 21:213-251.

Whittaker, R. H. 1975. Communities and ecosystems. Second edition. MacMillan, New York, New York, USA.

Williams, C. B. 1964. Patterns in the balance of nature. Academic Press, London, UK.

Williams, M. R. 1996. Species-area curves: the need to include zeroes. Global Ecology and Biogeography Letters 5: 91-93.

Williamson, M. 1988. Relationship of species number to area, distance and other variables. Pages 91-115 in A. A. Myers and P. S. Giller, editors. Analytical biogeography. Chapman and Hall, London, UK.

Williamson, M. 2003. Species-area relationships at small scales in continuum vegetation. Journal of Ecology 91: 904-907.

Williamson, M., K. J. Gaston, and W. M. Lonsdale. 2001. The species-area relationship does not have an asymptote! Journal of Biogeography 28:827-830.

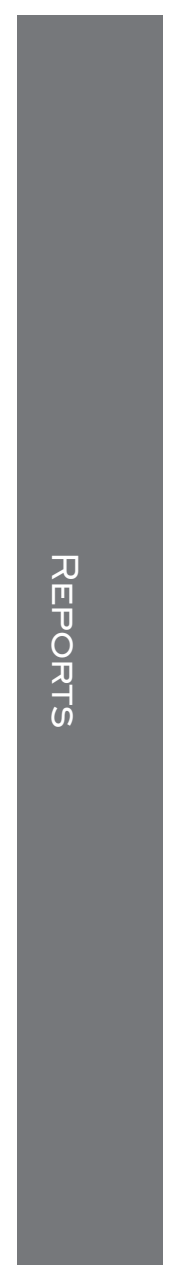

A figure presenting plot locations from the Carolina Vegetation Survey is available in ESA's Electronic Data Archive: Ecological Archives E086-064-A1.

\section{SUPPLEMENT}

Multiscalar richness data for 1472 Carolina Vegetation Survey plots are available in ESA's Electronic Data Archive: Ecological Archives E086-064-S1. 